\title{
Towards realistic laparoscopic image generation using image-domain translation
}

\author{
Aldo Marzullo ${ }^{\mathrm{a}}$, Sara Moccia ${ }^{\mathrm{b}, \mathrm{c}}$, Michele Catellani $^{\mathrm{d}}$, Francesco Calimeri ${ }^{\mathrm{a}}$, Elena De Momi ${ }^{\mathrm{e}}$ \\ ${ }^{a}$ Department of Mathematics and Computer Science, University of Calabria, Rende, Italy \\ ${ }^{b}$ Department of Information Engineering, Unviersità Politecnica delle Marche, Ancona, Italy \\ ${ }^{c}$ Department of Advanced Robotics, Istituto Italiano di Tecnologia, Genoa, Italy \\ ${ }^{d}$ Department of urology, European Institute of Oncology (IEO), IRCCS, Milan, Italy \\ ${ }^{e}$ Department of Electronics, Information and Bioengineering, Politecnico di Milano, Milan, Italy
}

\section{A R T I C L E I N F O}

Article history:

Keywords: Generative Adversarial Networks, Minimally Invasive Surgery, Image translation, Data Augmentation

\begin{abstract}
A B S T R A C T
Background and Objectives Over the last decade, Deep Learning (DL) has revolutionized data analysis in many areas, including medical imaging. However, there is a bottleneck in the advancement of DL in the surgery field, which can be seen in a shortage of large-scale data, which in turn may be attributed to the lack of a structured and standardized methodology for storing and analyzing surgical images in clinical centres. Furthermore, accurate annotations manually added are expensive and time consuming. A great help can come from the synthesis of artificial images; in this context, in the latest years, the use of Generative Adversarial Neural Networks (GANs) achieved promising results in obtaining photo-realistic images. Methods In this study, a method for Minimally Invasive Surgery (MIS) image synthesis is proposed. To this aim, the generative adversarial network pix2pix is trained to generate paired annotated MIS images by transforming rough segmentation of surgical instruments and tissues into realistic images. An additional regularization term was added to the original optimization problem, in order to enhance realism of surgical tools with respect to the background. Results Quantitative and qualitative (i.e., human-based) evaluations of generated images have been carried out in order to assess the effectiveness of the method. Conclusions Experimental results show that the proposed method is actually able to translate MIS segmentations to realistic MIS images, which can in turn be used to augment existing data sets and help at overcoming the lack of useful images; this allows physicians and algorithms to take advantage from new annotated instances for their training.
\end{abstract}

(C) 2020 Elsevier B. V. All rights reserved.

\section{Introduction}

Minimally invasive surgery (MIS) is currently the elective treatment for many procedures, such as nephrectomy and prostatectomy. By relying on small incisions on patient abdomen, through which surgical tools and endoscope are inserted, MIS attenuates some of the drawbacks of open surgery, such as prolonged patient hospitalization and recovery time [1].

e-mail: marzullo@mat.unical.it (Aldo Marzullo)
Nevertheless, MIS suffers from some drawbacks in terms of reduced field of view on the surgical site, which may hamper surgeon context awareness, and restricted freedom of movement for surgical action [2]. The surgical data science (SDS) community has been more and more focusing on developing solutions for Computer Assisted Interventions (CAI), with the final goal of increasing context awareness and providing decision support to surgeons [3]. Surgical phase recognition [4], 3-D reconstruction of soft tissues [5], tissue classification [6] and surgical tool segmentation and pose estimation [7] are among the main challenges of SDS. To address these challenges, while tackling 
the high variability encoded in the laparoscopic images, CAI methods commonly rely on deep learning (DL) [8]. The development of effective DL algorithms requires large annotated datasets, which are currently not available in this field, despite laudable initiatives promoted by some scientific communities (e.g., MICCAI $]^{1}$ and ISB ${ }^{2}$. Manual annotation has to be performed, which consists of time-consuming and tedious procedures; indeed, even though it has been shown that untrained anonymous individuals from online communities can generate training data of expert quality, obtaining manual annotation still remains a challenging task [9].

Data augmentation using affine transformations (e.g., rotation and scaling) is often used to try to tackle the small size of annotated datasets in several scenarios. Nonetheless, this approach presents known drawbacks, such as very limited diversities in existing data, especially when a small dataset is processed for augmentation [10]. Researchers in other fields proposed to artificially generate unseen images, while automatically providing semantic label-map annotation, using generative adversarial networks (GANs). GANs have been proven to be able to estimate the underlined distribution of data when dealing with very complicated data structures, and learn to replicate meaningful samples [11, 12, 13].

In this work, we specifically address the problem of generating realistic MIS images with surgical tools in the field of view, using the surgical-tool semantic label maps as constraint for the GAN generation process. This concept, in the literature referred to as image-to-image translation problem, is widely explored for synthesizing photos from label maps, reconstructing objects from edge maps, and colorizing images. A way to address image-to-image translation problems is to use conditional GANs (cGANs), which leverage standard GANs in a conditional setting [14]. In the herein addressed scenario, cGANs allow one to generate realistic MIS images, while having the corresponding surgical-tool label masks available. This has great potential, as the number of currently available datasets of surgical instruments in MIS is limited (such as ENDOVIS Grand Challeng $\varepsilon^{3}$, despite a growing number of research works have been published on the topic of instrument-tool analysis during MIS in the last few years [15, 7, 16].

\subsection{Contribution}

In order to tackle the issues described above, we propose to train a pix2pix cGAN [14] to translate semantic segmentation label maps of surgical tools to realistic MIS images with surgical tools in the field of view. To the best of our knowledge, although cGANs have been used for tasks in the MIS field, previous approaches only marginally involved surgical tools in the field of view, being more focused on colonoscopy data (see Sec. 22). As previously reported in the literature [17], cGANs allow to optimize image-translation and RGB-image prediction

\footnotetext{
http://www.miccai.org/

2 http://2020.biomedicalimaging.org/

https://endovis.grand-challenge.org/
}

(a)

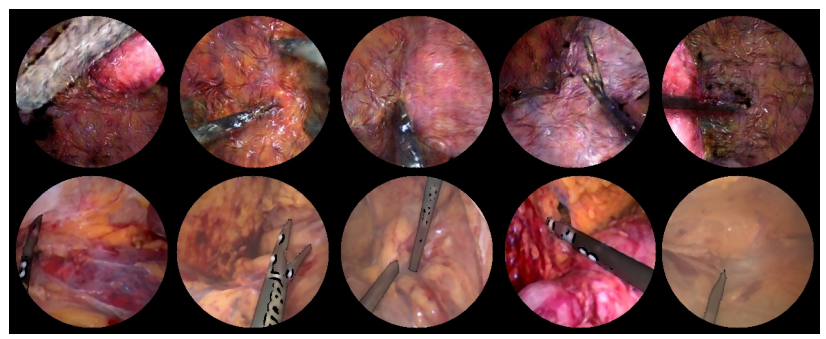

Fig. 1. Samples from the (a) generated and (b) real MIS images.

simultaneously, while remaining independent of camera, lighting and patient characteristics. In order to further condition the image-to-image translation problem, in this work we add a rough tissue-label map to the surgical-tool label map. This is done to tackle the high variability encoded in MIS images, in terms of noise, blur, illumination levels, tissues in the field of view (with different shape and texture), presence of occlusions and surgical tool pose, size and shape (as shown in Fig. 11). It is worth noting that obtaining such rough label map for abdominal tissues is a trivial task, and state- of-the-art algorithms can be used for the purpose (e.g., [6]).

The original pix2pix loss function was here modified to improve the realism of surgical tools by adding an additional regularization parameter, which leverages the pix 2pix generator to preserve the high-level features of surgical tools (e.g., sharp borders). The generated images were evaluated both analytically and by means of a custom web platform, where surgeons and SDS experts were asked to examine images and try to correctly identify if they were real or "fake".

The remainder of the paper is structured as follows. In Section 2 we survey relevant work in the literature, focusing on cGAN applications for endoscopic images. Section 3 provides a detailed description of the proposed approach; results are presented in Section 4 and discussed in Section 5 . Eventually, Section 6 draws our conclusions and summarizes the main contribution of the present work.

\section{Related work on cGANs in endoscopy}

The GAN framework has gained growing interest in computer vision and medical imaging as a more reliable approach to generate realistic training images rather than using synthetically augmented datasets. Applications include magnetic-resonance images generation [18, 11], liver-lesion classification [19], and pathology-progress simulation [20].

Recently, research in the field of GANs is focusing more and more on image-to-image translation problem, with the goal to generate new, unseen images as a combination of the content of an image and the style of another. Among those methods, the pix2pix network [14] received particular attention and has been used a wide range of sectors, including the biomedical domain [17]. The pix2pix is a deep learning model that solves the image-translation problem using a cGAN to learn a function to map the input image to the output one. The framework is composed by two main components, the generator and the discriminator. The generator is trained to transform the input image to 
the target one, while the discriminator measures the output and target similarity to encourage the generator to create a plausible translation. Another approach proposed for image-translation problems is CycleGAN [21], where two generator and two discriminator models are introduced for modeling both the direct and inverse translation mapping. In the specific context of laparoscopic images, both models have achieved remarkable results.

Focusing on the endoscopy domain, Rau et al. [17] used the pix2pix method to transform endoscopic images into depth maps, to compensate for the lack of labelled training data for deep-learning algorithms for depth estimation. Mathew et al. [22] presented a deep-learning framework, called Extended and Directional CycleGAN, for image-to-image translation between optical and virtual colonoscopy, with the final foal of increasing the size of optical colonoscopy data. Similarly, Oda et al. [23] used CycleGAN for generating optical colonoscopy data from virtual ones obtained from computerized tomography scans. CycleGAN has also being used by Esteban et al. [24], to translate bronchoscopic intra-operative videos to virtual bronchoscopies.

The majority of the approaches in the literature are focused on generating images with only one structure in the field of view (e.g., bronchi or colon). With respect to those endoscopic images, MIS images present higher variability in terms of tissues in the field of view, surgical tools shape, size and pose, and illumination level. Images acquired during MIS often present high noise level, blur, specularities, as well as presence of smoke and blur in the field of view. All this aspects pose challenges to the generation of realistic images. Hence, as the best of our knowledge, this work is the first attempt which investigates the problem of translating semantic label maps into realistic MIS images.

CycleGAN has been observed to work well on tasks that involve color or texture changes, like virtual to optical colonoscopy. However, for tasks that require substantial geometric changes to the image, such as cat-to-dog translations, CycleGAN usually fails [21]. Hence, in this work, the pix 2pix method is used to produce a mapping between semantic label maps and new, unseen, RGB MIS images.

\section{Materials and Methods}

In the following, we report a detailed description of background techniques and methods, along with details on the herein proposed approach.

\subsection{Dataset Description and Semantic Label Map Preparation}

The EndoVis Dataset from the EndoVis Intrument Segmentation and Tracking Challenge 2017 $7^{4}$ was used for this study. Sample images from the dataset are shown in Fig. 11(b). The dataset is made of $402 \mathrm{D}$ in-vivo images (image size $=720 \times 576$ pixels) from 4 laparoscopic colorectal surgeries, for a total of 160 images with the corresponding surgical-tool label map.

\footnotetext{
${ }^{4}$ https://endovissub-instrument.grand-challenge.org/Data/
}

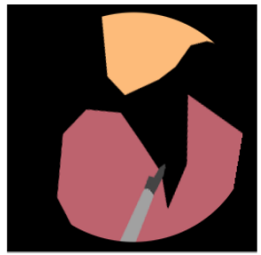

segmentation

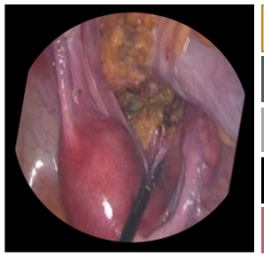

real image
Fig. 2. Schematic representation of the semantic label map used to generate realistic MIS images. The label map includes accurate surgical tool annotation and rough annotation of anatomic structures (i.e., fat and abdominal organs).

Each pixel is labelled as either background, shaft and manipulator.

Rough manual annotation of tissues was added to the original EndoVis label maps in order to explicitly leverage pix2pix learning the variability encoded in abdominal tissues. We empirically observed that having such a rough annotation allowed to increase the realism of generated images. Tissues were roughly annotated considering two classes, namely abdominal organs and fat. A sample of the resulting semantic label map, with both surgical tools and abdominal tissues, is shown in Fig. 2.

We also applied geometrical and morphological data augmentation (i.e., rotation, scaling, and shifting) to increase the number of training instances by a factor of 10 .

\subsection{Conditional Generative Adversarial Networks}

As introduced in Sec. 1, GANs are a class of generative models that learn the probability distribution over a dataset [25]. The standard GAN implementation can be described as a zerosum game, in which two networks, the Generator $(G)$ and Discriminator $(D)$, compete to one another to maximize its own payoff.

In this adversarial process, the two networks are trained simultaneously: the Generator network produces samples $x=G\left(z \mid \theta^{(g)}\right)$, where $\theta^{(g)}$ refers to the parameters of $G$, and $z$ is a random input noise (samples from a uniform distribution). The Discriminator network estimates $D\left(x \mid \theta^{(d)}\right)$, which refers to the probability that $x$ is a real training example, rather than an artificial sample drawn from the model.

Formally, the training objective for a GAN can be described as:

$$
G^{\star}=\arg \min _{G} \max _{D} \mathcal{L}_{G A N}(G, D)
$$

where:

$$
L_{G A N}(G, D)=E_{x}[\log D(x)]+E_{x, z}[\log (1-D(x, G(x, z))]
$$

The cGANs [21, 26, 14] extend the GANs by conditioning the generator and critic on prior information $(c)$. The training objective for a Conditional GAN then becomes:

$$
\begin{array}{r}
L_{c G A N}(G, D):=E_{x, c}[\log D(x, c)]+ \\
E_{x, z}[\log (1-D(x, G(x, z))]
\end{array}
$$


Several researchers have pointed out the benefits of combining the cGAN objective with a more traditional loss, such as the $L_{1}$ distance [26, 14]. This is formally expressed by:

$$
L_{1}(G)=E_{x, c, z}\left[\|c-G(x, z)\|_{1}\right]
$$

This objective can be used to train to produce outputs that look realistic and as close as possible to the real label, that is, it regularizes the generator model to output images that are a plausible translation of the source image. The final objective is then:

$$
G^{\star}=\arg \min _{G} \max _{D} L_{c G A N}(G, D)+\lambda \mathcal{L}_{1}(G)
$$

where $\lambda$ is a regularization parameter.

\section{3. cGAN for laparoscopic image generation}

In this work, we take advantage of a pix2pix cGAN [14] to translate a semantic label map relevant to tissue and instruments in realistic, synthetic, laparoscopic images. The pix2pix architecture is illustrated in Fig. 3, and described in Table 1 (generator) and Table 2 (discriminator).

In pix2pix, the discriminator is a deep CNN that performs conditional-image classification. The discriminator is a CNN that is run at patch level. The difference between the pix 2pix discriminator and standard discriminators is that, instead of producing output as single scalar vector, the pix2pix discriminator generates an $N \times N$ array, when $N \times N$ is the patch size. The average prediction of all patches is used to classify the whole image as real or fake.

The generator follows the U-Net encoder-decoder network architecture, with long skip connections between mirrored layers in the encoder and decoder stacks for recovering the resolution lost in the encoder path [27]. The network incorporates down-scaling on multiple levels to learn different degree of details, from a general representation to more local feature representations. Such architecture has been shown produce excellent results in several relevant scenarios, including medical image segmentation and translation [17, 14, 27]

The generator is trained on pairs $(x, c)$, where $x$ is the semantic label map with tissues and surgical tools and $c$ is the corresponding RGB endoscopic image. The random noise vector $z$ of Eq. 5 is not provided as input, instead, it is simulated through dropout layers with probability 0.5 during both training and inference. Such an approach has been proven to give comparable results, while simplifying the overall implementation [14].

The endoscopic images we aim to simulate in this study can be schematically decomposed in two semantic sets: surgical tools and abdominal tissues. However, because of several differences in light and shapes, learning to simulate such hierarchy could not be a trivial task, resulting in blurred and non-realistic surgical-tool boundaries. In this work, to leverage a more effective image-to-image translation, we explicitly penalise differences between the true and generated surgical tools. Let $t$ be the semantic label map of the surgical tools in an image, the loss function of the cGAN (Eq. 5) was modified by adding a further regularization term:

$$
L_{t}(G)=E_{x, c, t, z}\left[\|c t-G(x, z) t\|_{1}\right]
$$

\begin{tabular}{|c|c|c|c|}
\hline Id & Layer (type) & Output Shape & Connected to \\
\hline 1 & Input & $256 \times 256 \times 3$ & \\
\hline 2 & 2DConv & $128 \times 128 \times 64$ & 1 \\
\hline $2 \mathrm{a}$ & Leaky ReLU & $128 \times 128 \times 64$ & 2 \\
\hline 3 & 2DConv & $64 \times 64 \times 128$ & $2 a$ \\
\hline $3 a$ & Batch Norm & $64 \times 64 \times 128$ & 3 \\
\hline $3 b$ & Leaky ReLU & $64 \times 64 \times 128$ & $3 a$ \\
\hline 4 & 2DConv & $32 \times 32 \times 256$ & $3 b$ \\
\hline $4 a$ & Batch Norm & $32 \times 32 \times 256$ & 4 \\
\hline $4 \mathrm{~b}$ & Leaky ReLU & $32 \times 32 \times 256$ & $4 a$ \\
\hline 5 & 2DConv & $16 \times 16 \times 512$ & $4 \mathrm{~b}$ \\
\hline $5 \mathrm{a}$ & Batch Norm & $16 \times 16 \times 512$ & 5 \\
\hline $5 b$ & Leaky ReLU & $16 \times 16 \times 512$ & $5 \mathrm{a}$ \\
\hline 6 & 2DConv & $8 \times 8 \times 512$ & $5 b$ \\
\hline $6 a$ & Batch Norm & $8 \times 8 \times 512$ & 6 \\
\hline $6 b$ & Leaky ReLU & $8 \times 8 \times 512$ & $6 a$ \\
\hline 7 & 2DConv & $4 \times 4 \times 512$ & $6 b$ \\
\hline $7 a$ & Batch Norm & $4 \times 4 \times 512$ & 7 \\
\hline $7 b$ & Leaky ReLU & $4 \times 4 \times 512$ & $7 \mathrm{a}$ \\
\hline 7 & 2DConv & $2 \times 2 \times 512$ & $6 b$ \\
\hline $7 \mathrm{a}$ & Batch Norm & $2 \times 2 \times 512$ & 7 \\
\hline $7 b$ & Leaky ReLU & $2 \times 2 \times 512$ & $7 \mathrm{a}$ \\
\hline 8 & 2DConv & $1 \times 1 \times 512$ & $7 b$ \\
\hline $8 b$ & Leaky ReLU & $1 \times 1 \times 512$ & 8 \\
\hline 9 & 2DConvT & $2 \times 2 \times 512$ & $8 b$ \\
\hline $9 a$ & Batch Norm & $2 \times 2 \times 512$ & 9 \\
\hline $9 b$ & Dropout & $2 \times 2 \times 512$ & $9 a$ \\
\hline $9 \mathrm{c}$ & Concatenate & $2 \times 2 \times 1024$ & $9 b, 7 b$ \\
\hline $9 \mathrm{~d}$ & Leaky ReLU & $2 \times 2 \times 1024$ & $9 \mathrm{c}$ \\
\hline 10 & 2DConvT & $4 \times 4 \times 512$ & $9 \mathrm{~b}$ \\
\hline $10 \mathrm{a}$ & Batch Norm & $4 \times 4 \times 512$ & 10 \\
\hline $10 \mathrm{~b}$ & Dropout & $4 \times 4 \times 512$ & $10 \mathrm{a}$ \\
\hline $10 \mathrm{c}$ & Concatenate & $4 \times 4 \times 1024$ & $10 \mathrm{~b}, 6 \mathrm{~b}$ \\
\hline $10 \mathrm{~d}$ & Leaky ReLU & $4 \times 4 \times 1024$ & $10 \mathrm{c}$ \\
\hline 11 & 2DConvT & $8 \times 8 \times 512$ & $10 \mathrm{~b}$ \\
\hline $11 \mathrm{a}$ & Batch Norm & $8 \times 8 \times 512$ & 11 \\
\hline $11 \mathrm{~b}$ & Dropout & $8 \times 8 \times 512$ & $11 \mathrm{a}$ \\
\hline $11 \mathrm{c}$ & Concatenate & $8 \times 8 \times 1024$ & $11 b, 5 b$ \\
\hline $11 \mathrm{~d}$ & Leaky ReLU & $8 \times 8 \times 1024$ & $11 \mathrm{c}$ \\
\hline 12 & 2DConvT & $16 \times 16 \times 512$ & $11 \mathrm{~b}$ \\
\hline $12 \mathrm{a}$ & Batch Norm & $16 \times 16 \times 512$ & 12 \\
\hline $12 b$ & Concatenate & $16 \times 16 \times 1024$ & $12 a, 4 b$ \\
\hline $12 \mathrm{c}$ & Leaky ReLU & $16 \times 16 \times 1024$ & $12 \mathrm{~b}$ \\
\hline 13 & 2DConvT & $32 \times 32 \times 256$ & $12 b$ \\
\hline $13 \mathrm{a}$ & Batch Norm & $32 \times 32 \times 256$ & 13 \\
\hline $13 b$ & Concatenate & $32 \times 32 \times 256$ & $13 a, 3 b$ \\
\hline $13 \mathrm{c}$ & Leaky ReLU & $32 \times 32 \times 512$ & $13 b$ \\
\hline 14 & 2DConvT & $64 \times 64 \times 128$ & $13 \mathrm{~b}$ \\
\hline $14 \mathrm{a}$ & Batch Norm & $64 \times 64 \times 128$ & 14 \\
\hline $14 \mathrm{~b}$ & Concatenate & $64 \times 64 \times 128$ & $14 a, 2 b$ \\
\hline $14 \mathrm{c}$ & Leaky ReLU & $64 \times 64 \times 256$ & $14 \mathrm{~b}$ \\
\hline 15 & 2DConvT & $128 \times 128 \times 64$ & $14 \mathrm{~b}$ \\
\hline $15 \mathrm{a}$ & Batch Norm & $128 \times 128 \times 64$ & 15 \\
\hline $15 b$ & Concatenate & $128 \times 128 \times 64$ & $15 \mathrm{a}, 1 \mathrm{~b}$ \\
\hline $15 c$ & Leaky ReLU & $128 \times 128 \times 128$ & $15 b$ \\
\hline 16 & 2DConvT & $256 \times 256 \times 3$ & $15 \mathrm{c}$ \\
\hline 16 & Tanh & $256 \times 256 \times 3$ & 16 \\
\hline
\end{tabular}

Table 1. Architecture of the generator network 

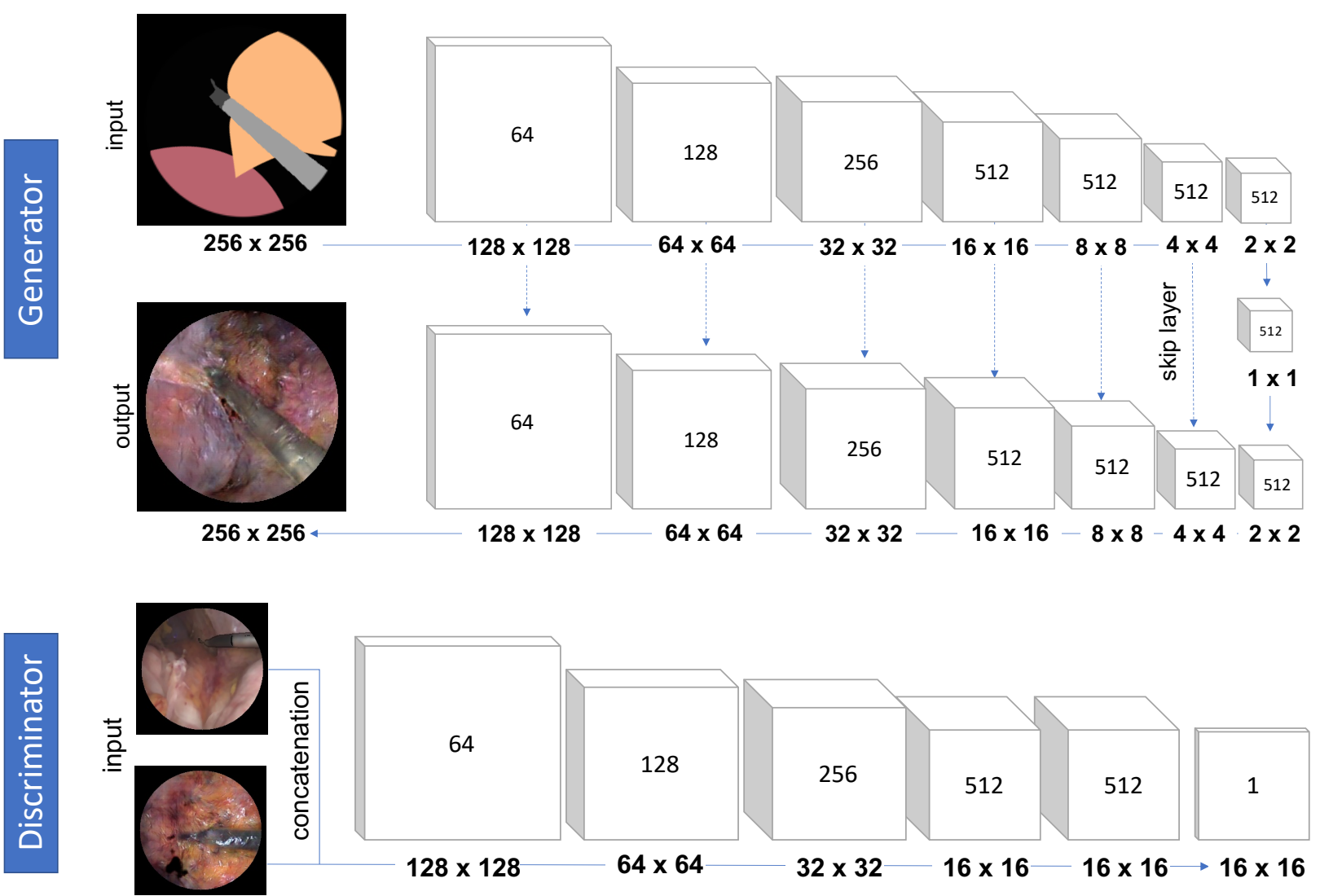

Fig. 3. Proposed pix2pix framework for laparoscopic-image generation. The (top) generator and (bottom) discriminator networks are shown. The featuremap size and the number of channels is reported, too.

Table 2. Architecture of the discriminator network

\begin{tabular}{llcc}
\hline Id & Layer (type) & Output Shape & Connected to \\
\hline $1 \mathrm{a}$ & Input & $256 \times 256 \times 3$ & \\
$1 \mathrm{~b}$ & Input & $256 \times 256 \times 3$ & \\
\hline $1 \mathrm{c}$ & Concatenate & $256 \times 256 \times 6$ & $1 \mathrm{a}, 1 \mathrm{~b}$ \\
\hline 2 & 2DConv & $128 \times 128 \times 64$ & $1 \mathrm{c}$ \\
$2 \mathrm{a}$ & Leaky ReLU & $128 \times 128 \times 64$ & 2 \\
\hline 3 & 2DConv & $64 \times 64 \times 128$ & $2 \mathrm{a}$ \\
$3 \mathrm{a}$ & Batch Norm & $64 \times 64 \times 128$ & 3 \\
$3 \mathrm{~b}$ & Leaky ReLU & $64 \times 64 \times 128$ & $3 \mathrm{a}$ \\
\hline 4 & 2DConv & $32 \times 32 \times 256$ & $3 \mathrm{~b}$ \\
$4 \mathrm{a}$ & Batch Norm & $32 \times 32 \times 256$ & 4 \\
$4 \mathrm{~b}$ & Leaky ReLU & $32 \times 32 \times 256$ & $4 \mathrm{a}$ \\
\hline 5 & 2DConv & $16 \times 16 \times 512$ & $4 \mathrm{~b}$ \\
$5 \mathrm{a}$ & Batch Norm & $16 \times 16 \times 512$ & 5 \\
$5 \mathrm{~b}$ & Leaky ReLU & $16 \times 16 \times 512$ & $5 \mathrm{a}$ \\
\hline 6 & 2DConv & $16 \times 16 \times 512$ & $5 \mathrm{~b}$ \\
$6 \mathrm{a}$ & Batch Norm & $16 \times 16 \times 512$ & 6 \\
$6 \mathrm{~b}$ & Leaky ReLU & $16 \times 16 \times 512$ & $6 \mathrm{a}$ \\
\hline 7 & 2DConv & $16 \times 16 \times 1$ & $6 \mathrm{~b}$ \\
$7 \mathrm{~b}$ & Leaky ReLU & $16 \times 16 \times 1$ & 7 \\
\hline & & &
\end{tabular}

Our objective loss was then defined as:

$$
G^{t}=\arg \min _{G} \max _{D} L_{C G A N}(G, D)+\lambda_{1} L_{1}(G)+\lambda_{2} L_{t}(G)
$$

where $\lambda_{1}$ is the overall regularizing weight, while $\lambda_{2}$ is the regularizing weight specific to the surgical tools.

\subsection{Experimental settings}

The pix2pix architecture was implemented and trained using Keras. The Adam optimizer was chosen with a learning rate of $2 \times 10^{-3}$ for both the generator and discriminator. We empirically set the $L_{1}\left(\lambda_{1}=50\right)$ and $L_{t}\left(\lambda_{2}=100\right)$ regularization parameters to reflect the fact that the semantic label map was accurate for the surgical tools, while it was rough for the abdominal tissues. Patch size in the discriminator was set to $70 \times 70$ pixels. Images were scaled to $256 \times 256$ pixels for memory constraints. The images were normalized in the range $[-1,1]$. We trained the pix2pix for 300 epochs using a batch size of 1 . We stopped the training when satisfying visual results were achieved on a small test set of 10 , unseen, images (this happened after $\sim 100$ epochs). Training took about 6 hours on a NVIDIA Quadro P6000 GPU.

\subsection{Experimental Protocol}

Currently, there is no established consensus, in the research community, on the best way to evaluate GANs [25, 11]. Indeed, 
it is not trivial to obtain the definition of precise mathematical rules allowing one to tell whether an image is eligible for belonging to a given group or not. For instance, considering probability distributions between pixels or regions of the image by using statistical approaches might not be enough, as geometrical relationships between objects in the image should be taken into account. For these reasons, in this study we tested our approach by means of both quantitative measurements and qualitative visual assessments by surgeons and SDS experts over the generated laparoscopic images.

\subsubsection{Human evaluation of generated images}

A web platform was build, where physicians and experts were called to distinguish between real and synthetic endoscopic images. In order to generate completely unseen synthetic MIS images, a proper Python script was defined to generate semantic-label maps with tissues and surgical tools. The idea was to randomly generate polygons (representing the tissues and tools) over a black background. The trained pix 2 pix was used to translate these semantic-label maps into synthetic MIS images.

Two sets of 160 images (160 synthetic and 160 real) were considered. More precisely, we iteratively showed to each judge 20 pairs consisting of a real image and a generated one, extracted from the two sets with equal probability. The judge has to tell which one was the fake and which one was the real image. During each of the 20 trials, true positive $(T P)$, true negative $(T N)$, false positive $(F P)$ and false negative $(F N)$ and the number of correct response were collected, where positive and negative refer to the generated and original images, respectively.

A total of 54 tests were collected by different users, divided in four categories (17 naive users, 19 non-expert surgeons, 13 expert surgeons, 5 technical developers). In order to guarantee a fair comparison, a postprocessing step (Gaussian blur) was added to the real images, in order to make their visual quality comparable to that of synthetic images. This was done to avoid the evaluators focusing on high-level details (e.g. instrument brand) to spot real images.

The one-way ANOVA test was performed to assess if any statistical difference between the groups of evaluators existed. Group normality and homoscedasticity assumptions were evaluated by means of the Shapiro-Wilk and Bartlett's test, respectively ( significance level $=5 \%$ ).

\subsubsection{Quantitative image-quality assessment}

In order to evaluate the similarity between the two groups (i.e., the real and generated images), the distributions of real and synthetic datasets were estimated by means of the Kernel Density Estimation (KDE) function, and their likelihoods were compared. Similar approaches were already applied in the context of GANs in [11] and [25]. In our approach, we computed the similarity between the two datasets estimating their distribution by means of the Kernel Density function, so that similar datasets should be represented by similar distributions. More in detail, the probability of the synthetic data is estimated by fitting a Gaussian Parzen window to the generated samples and

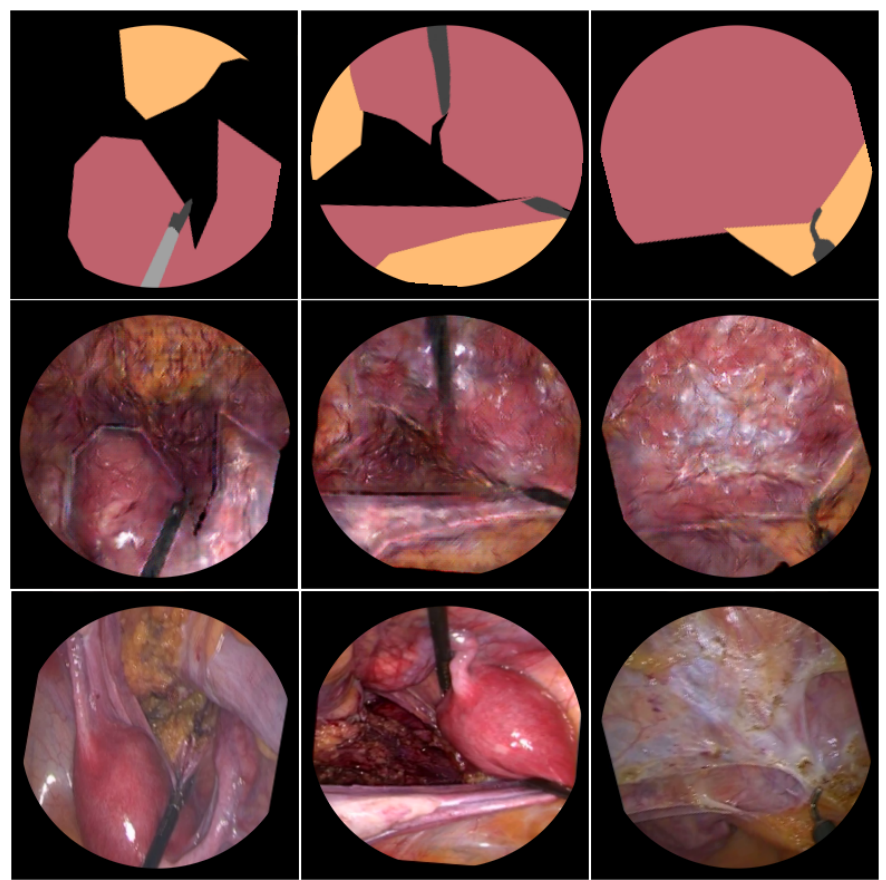

Fig. 4. Sample from the image translation results. Starting from the top row, the input semantic label map, the corresponding translation and the ground truth are shown.

reports the likelihood under this distribution. The bandwidth of the Gaussians is obtained by cross-validating the training data. In addition, for a visual comparison of the two groups, Principal Component Analysis (PCA) embedding representation of real and synthetic images was computed [28]. Several embedding dimension ( $d$, from 10 to 100 with a 10 -unit increments) were analysed without noticing any significant visual difference. Thus, $d=100$ was used for the experiments.

\subsubsection{Surgical tool segmentation}

In order to quantitatively assess the informative content of the generated images, we evaluated whether the proposed method can serve as a data augmentation method for surgical-tool segmentation tasks. A total of 1600 images were generated by means of the proposed approach and used to train a standard UNet architecture [27]. The network was trained for 50 epochs using binary cross-entropy as loss function and Adam optimizer (learning rate $=0.0001$, batch size $=16$ ). The model was implemented in Keras and trained using a NVIDIA Quadro P6000 GPU. After training, the best model was chosen as the one that minimized the loss on the validation set $30 \%$ of the whole dataset).

The model was finally tested on 40 images from the original MIS dataset. We calculate five evaluation indexes respectively: Søresen Dice Coefficient (Dice), Jaccard Similarity (Jaccard), Precision (Precision), Recall (Recall) and F1-score (F1):

$$
\begin{gathered}
\text { Dice }=\frac{2 T P}{2 T P+F N+F P} \\
\text { Jaccard }=\frac{T P}{T P+F N+F P}
\end{gathered}
$$




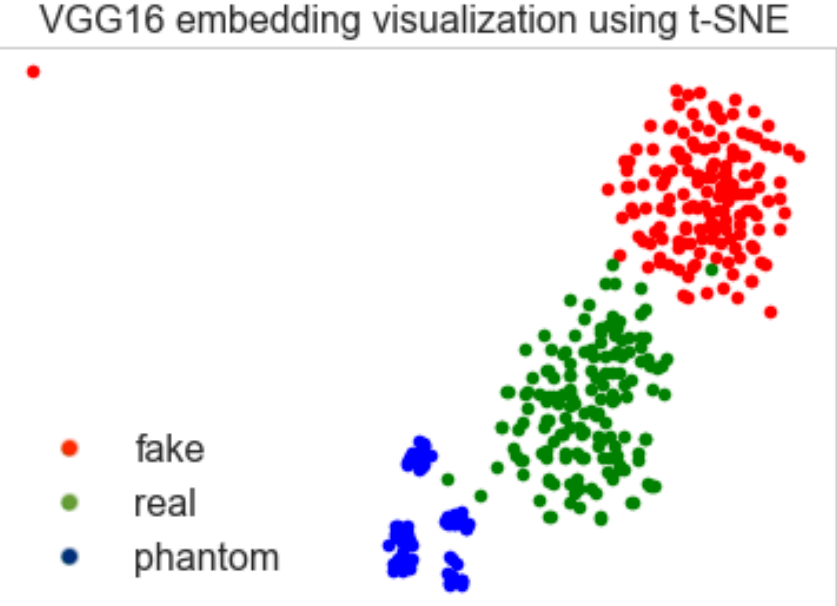

Fig. 5. Bi-dimensional Principal Component Analysis (PCA) embedding representation of real and synthetic images.

$$
\begin{gathered}
\text { Precision }=\frac{T P}{T P+F P} \\
\text { Recall }=\frac{T P}{T P+F N} \\
F 1=\frac{2 \cdot \text { Precision } \cdot \text { Recall }}{\text { Precision }+ \text { Recall }}
\end{gathered}
$$

It is important to point out that the main goal of the study was not to achieve high segmentation performance; rather, we wanted to evaluate the informative content of the artificially generated images. For this reason, no further parameter tuning was performed to improve segmentation results.

\section{Results}

Samples from the generated images starting from the semantic label map with accurate surgical-tool segmentation and rough tissue segmentation can be seen in Figure 4 . The average results from human evaluation are reported in Table 3 No differences were found between the assessor groups, except for the technical developers. The difficulty to differentiate between the real and generated images is well underlined by the low values of correct instances guessed by humans in the tests. Expert surgeons achieved the highest results. However, a similar performance was achieved by both naive users and non-expert surgeons. It is interesting to observe the high score achieved by the technical developer, which were able to spot the typical artefacts of synthetic images produced by GANs.

Values of 33.5765 and 33.5791 were obtained respectively by comparing the log-likelihoods for the 160 true and 160 synthetic data. This result suggests that the two groups are similar but not identical. Such similarity can be observed by the visual representation of the first two dimensions of the embedding $(d$ $=100$ ) illustrated in Figure 5 . The two distributions do not overlap, suggesting that the pix-to-pix did not replicate images from the original set.

Average U-Net segmentation results are reported in Table 4. the synthetic images provided high enough informative content to let the model generalize on real instances, achieving encouraging results.

\section{Discussion}

In this work, we presented a deep-learning approach based on cGANs for generating realistic laparoscopic images with surgical instruments. Because annotated MIS datasets are difficult to obtain, due to high costs in terms of money and time required to perform the annotation, the proposed approach provides an effective method to augment MIS endoscopic datasets, with a view to generate SDS methods that rely on instrumenttool analysis. We took advantage of the pix2pix architecture for image-domain translation, incorporating instrument and (rough) tissue segmentation as priors. For pix 2 pix training, we exploited the publicly-available images of the EndoVis Intrument Segmentation and Tracking Challenge 2017.

Results were evaluated by means of quantitative and qualitative approaches. Fake images distribution was observed to be close to the real dataset, suggesting that our method was able to generate realistic contents without replicating the original images. In addition, we addressed the problem of image segmentation by training a well-known architecture used in the medical field, i.e. U-Net, using only synthetic images. Such simple training allowed to achieve promising segmentation results on real images, showing that the proposed method can be effectively applied for augmenting surgical datasets for segmentation tasks.

Naive users, surgeons and experts in GAN development were called to discriminate between real and newly generated images. In order to have a more detailed feedback, we also asked them to write down comments describing how did they tell the difference between real and synthetic images. Both naive users and surgeons were mostly unable to discriminate the images. It is worth to note that some of them raised concerns about the lower image quality with respect to that commonly available during their actual surgical practice, which, in fact, constitutes a limitation of our work. For a more fair comparison, indeed, we slightly modified real-images to avoid the evaluators focusing on high-frequency details (e.g. instrument brand) to discriminate between fake and real images. However, we found such processing not representing a limitation for the test, according to the high results and comments collected by technical developers and some of the surgeons. Technical developers (nonclinical), indeed, were significantly better at discriminating real and generated images. This may be probably attributed to their background, which made them able to to spot features relevant to GAN generation.

A second limitation of this study may be seen in the relatively small size of the training dataset. However, the lack of large and annotated publicly available datasets is a well known-problem in the SDS community [3]. This problem was addressed by performing linear transformations to the original available images. It is worth to note that methods for training image-toimage translation system that do not require paired examples exist. In this context, several experiments using CycleGAN [21] were performed but not reported in this paper. In particular, images generated by CycleGAN were observed to be only small 
Table 3. Results of the human evaluation in terms of True Positive $(T P)$, True Negative $(T N)$, False Positive $(F P)$, False Negative $(F N)$, number of correct response (Correct). Results are averaged across all users (all) and for each level of expertise ( \pm standard deviation).

\begin{tabular}{|c|c|c|c|c|c|c|c|c|}
\hline & count & No. Fake & No. Real & Correct & $T N$ & $T P$ & $F N$ & $F P$ \\
\hline overall & 54 & $10.19 \pm 2.47$ & $9.81 \pm 2.47$ & $7.94 \pm 5.37$ & $4.74 \pm 3.8$ & $3.2 \pm 3.1$ & $6.61 \pm 3.21$ & $5.44 \pm 3.14$ \\
\hline naive users & 17 & $10.12 \pm 2.6$ & $9.88 \pm 2.6$ & $7.29 \pm 5.51$ & $3.94 \pm 3.36$ & $3.35 \pm 3.18$ & $6.53 \pm 3.06$ & $6.18 \pm 3.21$ \\
\hline expert surgeon & 13 & $10.0 \pm 2.55$ & $10.0 \pm 2.55$ & $7.85 \pm 5.49$ & $5.15 \pm 4.18$ & $2.69 \pm 3.45$ & $7.31 \pm 2.9$ & $4.85 \pm 3.51$ \\
\hline technical developer* & 5 & $12.8 \pm 3.63$ & $7.2 \pm 3.63$ & $15.4 \pm 1.67$ & $10.6 \pm 3.71$ & $4.8 \pm 2.68$ & $2.4 \pm 2.61$ & $2.2 \pm 1.48$ \\
\hline
\end{tabular}

*number of correct responses significantly different from other groups

Table 4. Segmentation results in terms of Dice Coefficient (Dice), F1score ( $F 1)$, Jaccard Similarity (Jaccard), Precision (Precision) and Recall (Recall).

\begin{tabular}{lccccc}
\hline & Dice & $F 1$ & Jaccard & Precision & Recall \\
\hline mean & 0.71 & 0.97 & 0.59 & 0.81 & 0.68 \\
std & 0.22 & 0.02 & 0.22 & 0.18 & 0.26 \\
\hline
\end{tabular}

variations of the same image (mode collapse problem) and we were not able to overcome it with the current implementation. Such results were probably related to a common issue of $\mathrm{Cy}-$ cleGAN, which usually fails with tasks that require substantial geometric changes [21]. CycleGAN was successfully exploited in the majority of the approaches in the literature [22, 23, 24], which, however, focused on generating images with only one structure in the field of view (e.g., bronchi or colon). In this study, instead, we focused on images presenting higher variability, in terms of tissues in the field of view, surgical tools shape, size and pose, and illumination level, for which CycleGAN was challenging to train.

\section{Conclusions}

A laparoscopic image generation method was proposed in this study. The conditional generative adversarial network system pix2pix was exploited for generating synthetic images starting from an (accurate) surgical-tool and a (rough) abodminaltissue label map. Achieved results suggest that the method can be effectively used for augmenting surgical datasets for segmentation tasks. As future work is concerned, we aim at improving the quality of our results by exploiting advanced generative models such as pix2pixHD [29], also dealing with unpaired datasets. In addition, despite our analysis being focused on images with surgical tools, the proposed framework could be easily extended to other object classes. Hence, we plan to investigate the performance of the proposed approach also in other anatomical fields, e.g., for generating images with placenta membrane in fetoscopic images to support surgeons with context awareness [30].

\section{Acknowledgments}

This study did not need any ethical approval. The authors declare no competing interests. The study was partially supported by the European Commission and the Regione Calabria within the program POR Calabria FESR-FSE 2014/2020, topic "ICT e
Terziario Innovativo". We gratefully acknowledge the support of NVIDIA Corporation with the donation of the Quadro P6000 GPU used for this research.

\section{References}

[1] J. H. Palep, Robotic assisted minimally invasive surgery, Journal of Minimal Access Surgery 5 (1) (2009) 1-7.

[2] M. Cianchetti, A. Menciassi, Soft robots in surgery, in: Soft Robotics: Trends, Applications and Challenges, Springer, 2017, pp. 75-85.

[3] L. Maier-Hein, S. S. Vedula, S. Speidel, N. Navab, R. Kikinis, A. Park, M. Eisenmann, H. Feussner, G. Forestier, S. Giannarou, et al., Surgical data science for next-generation interventions, Nature Biomedical Engineering 1 (9) (2017) 691-696.

[4] D. Katić, C. Julliard, A.-L. Wekerle, H. Kenngott, B. P. Müller-Stich, R. Dillmann, S. Speidel, P. Jannin, B. Gibaud, LapOntoSPM: an ontology for laparoscopic surgeries and its application to surgical phase recognition, International Journal of Computer Assisted Radiology and Surgery 10 (9) (2015) 1427-1434.

[5] V. Penza, J. Ortiz, L. S. Mattos, A. Forgione, E. De Momi, Dense soft tissue $3 \mathrm{D}$ reconstruction refined with super-pixel segmentation for robotic abdominal surgery, International Journal of Computer Assisted Radiology and Surgery 11 (2) (2016) 197-206.

[6] S. Moccia, S. J. Wirkert, H. Kenngott, A. S. Vemuri, M. Apitz, B. Mayer, E. De Momi, L. S. Mattos, L. Maier-Hein, Uncertainty-aware organ classification for surgical data science applications in laparoscopy, IEEE Transactions on Biomedical Engineering 65 (11) (2018) 2649-2659.

[7] S. Bodenstedt, M. Allan, A. Agustinos, X. Du, L. Garcia-Peraza-Herrera, H. Kenngott, T. Kurmann, B. Müller-Stich, S. Ourselin, D. Pakhomov, et al., Comparative evaluation of instrument segmentation and tracking methods in minimally invasive surgery, arXiv preprint arXiv:1805.02475 (2018).

[8] G. Litjens, T. Kooi, B. E. Bejnordi, A. A. A. Setio, F. Ciompi, M. Ghafoorian, J. A. Van Der Laak, B. Van Ginneken, C. I. Sánchez, A survey on deep learning in medical image analysis, Medical Image Analysis 42 (2017) 60-88.

[9] L. Maier-Hein, T. Ross, J. Gröhl, B. Glocker, S. Bodenstedt, C. Stock, E. Heim, M. Götz, S. Wirkert, H. Kenngott, et al., Crowd-algorithm collaboration for large-scale endoscopic image annotation with confidence, in: International Conference on Medical Image Computing and Computer-Assisted Intervention, Springer, 2016, pp. 616-623.

[10] M. B. Lee, Y. H. Kim, K. R. Park, Conditional generative adversarial network-based data augmentation for enhancement of iris recognition accuracy, IEEE Access 7 (2019) 122134-122152.

[11] F. Calimeri, A. Marzullo, C. Stamile, G. Terracina, Biomedical data augmentation using generative adversarial neural networks, in: International Conference on Artificial Neural Networks, Springer, 2017, pp. 626-634.

[12] P. Andreini, S. Bonechi, M. Bianchini, A. Mecocci, F. Scarselli, Image generation by GAN and style transfer for agar plate image segmentation, Computer Methods and Programs in Biomedicine 184 (2020) 105268.

[13] T. Shen, C. Gou, F.-Y. Wang, Z. He, W. Chen, Learning from adversarial medical images for X-ray breast mass segmentation, Computer Methods and Programs in Biomedicine 180 (2019) 105012.

[14] P. Isola, J.-Y. Zhu, T. Zhou, A. A. Efros, Image-to-image translation with conditional adversarial networks, in: Proceedings of the IEEE Conference on Computer Vision and Pattern Recognition, 2017, pp. 1125-1134. 
[15] E. Colleoni, S. Moccia, X. Du, E. De Momi, D. Stoyanov, Deep learning based robotic tool detection and articulation estimation with spatiotemporal layers, IEEE Robotics and Automation Letters 4 (3) (2019) 2714-2721.

[16] M. Allan, S. Ourselin, D. J. Hawkes, J. D. Kelly, D. Stoyanov, 3-D pose estimation of articulated instruments in robotic minimally invasive surgery, IEEE Transactions on Medical Imaging 37 (5) (2018) 12041213.

[17] A. Rau, P. E. Edwards, O. F. Ahmad, P. Riordan, M. Janatka, L. B. Lovat, D. Stoyanov, Implicit domain adaptation with conditional generative adversarial networks for depth prediction in endoscopy, International Journal of Computer Assisted Radiology and Surgery 14 (7) (2019) 11671176.

[18] A. Radford, L. Metz, S. Chintala, Unsupervised representation learning with deep convolutional generative adversarial networks, arXiv preprint arXiv:1511.06434 (2015).

[19] M. Frid-Adar, I. Diamant, E. Klang, M. Amitai, J. Goldberger, H. Greenspan, GAN-based synthetic medical image augmentation for increased cnn performance in liver lesion classification, Neurocomputing 321 (2018) 321-331.

[20] H. Salehinejad, S. Valaee, T. Dowdell, E. Colak, J. Barfett, Generalization of deep neural networks for chest pathology classification in x-rays using generative adversarial networks, in: 2018 IEEE International Conference on Acoustics, Speech and Signal Processing, IEEE, 2018, pp. 990-994.

[21] J.-Y. Zhu, T. Park, P. Isola, A. A. Efros, Unpaired image-to-image translation using cycle-consistent adversarial networks, in: IEEE International Conference on Computer Vision, 2017, pp. 2223-2232.

[22] S. Mathew, S. Nadeem, S. Kumari, A. Kaufman, Augmenting colonoscopy using extended and directional CycleGAN for lossy image translation, arXiv preprint arXiv:2003.12473 (2020).

[23] M. Oda, K. Tanaka, H. Takabatake, M. Mori, H. Natori, K. Mori, Realistic endoscopic image generation method using virtual-to-real image-domain translation, Healthcare Technology Letters 6 (6) (2019) 214-219.

[24] A. Esteban-Lansaque, C. Sanchez, A. Borras, D. Gil, Augmentation of virtual endoscopic images with intra-operative data using content-nets, BioRxiv (2019) 681825.

[25] I. Goodfellow, J. Pouget-Abadie, M. Mirza, B. Xu, D. Warde-Farley, S. Ozair, A. Courville, Y. Bengio, Generative adversarial nets, in: Advances in Neural Information Processing Systems, 2014, pp. 2672-2680.

[26] M. Mirza, S. Osindero, Conditional generative adversarial nets, arXiv preprint arXiv:1411.1784 (2014).

[27] O. Ronneberger, P. Fischer, T. Brox, U-net: Convolutional networks for biomedical image segmentation, in: International Conference on Medical Image Computing and Computer-Assisted Intervention, Springer, 2015, pp. 234-241.

[28] H. Abdi, L. J. Williams, Principal component analysis, Wiley interdisciplinary Reviews: Computational Statistics 2 (4) (2010) 433-459.

[29] T.-C. Wang, M.-Y. Liu, J.-Y. Zhu, A. Tao, J. Kautz, B. Catanzaro, Highresolution image synthesis and semantic manipulation with conditional gans, in: IEEE Conference on Computer Vision and Pattern Recognition, 2018, pp. 8798-8807.

[30] A. Casella, S. Moccia, E. Frontoni, D. Paladini, E. De Momi, L. S. Mattos, Inter-foetus membrane segmentation for TTTS using adversarial networks, Annals of Biomedical Engineering 48 (2) (2020) 848-859. 